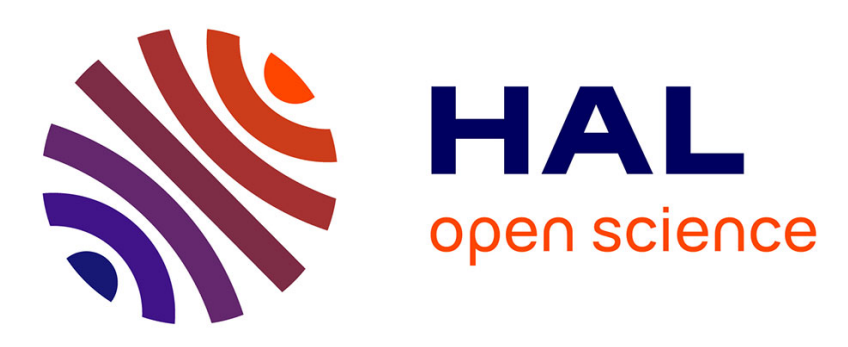

\title{
Modélisation fonctionnelle adaptée à l'analyse des risques en radiothérapie
}

Alexandre Reitz, Jean-François Pétin, Eric Levrat, Alain Noel, Delphine Aigle, Didier Peiffert, Pierre Graff

\section{- To cite this version:}

Alexandre Reitz, Jean-François Pétin, Eric Levrat, Alain Noel, Delphine Aigle, et al.. Modélisation fonctionnelle adaptée à l'analyse des risques en radiothérapie. Cancer/Radiothérapie, 2012, 16 (8), pp.653-660. 10.1016/j.canrad.2012.06.006 . hal-00727407

\section{HAL Id: hal-00727407 https://hal.science/hal-00727407}

Submitted on 4 Sep 2012

HAL is a multi-disciplinary open access archive for the deposit and dissemination of scientific research documents, whether they are published or not. The documents may come from teaching and research institutions in France or abroad, or from public or private research centers.
L'archive ouverte pluridisciplinaire HAL, est destinée au dépôt et à la diffusion de documents scientifiques de niveau recherche, publiés ou non, émanant des établissements d'enseignement et de recherche français ou étrangers, des laboratoires publics ou privés. 


\title{
Modélisation fonctionnelle adaptée à l'analyse des risques en radiothérapie
}

\author{
Functional modeling for risk analysis in radiotherapy
}

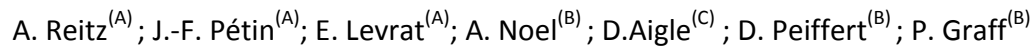

A. Université de Lorraine, Centre de Recherche en Automatique de Nancy (CRAN) - CNRS, UMR 7039, Campus sciences, BP 239, 54506 Vandœuvre-lès-Nancy Cedex, France

B. Service de Radiothérapie, Centre Alexis Vautrin, 54500 Vandœuvre-lès-Nancy, France

C. Service Qualité et Efficience, Centre Alexis Vautrin, 54500 Vandœuvre-lès-Nancy, France

Reitz Alexandre, Centre de recherche en Automatique de Nancy (CRAN), 2, avenue de la forêt de Haye, 54500 Vandœuvre-lès-Nancy, France

Tel : (+33)6.07.73.85.39

Mail : alexandre.reitz@univ-lorraine.fr;

\section{Résumé :}

Ce document présente une démarche originale de modélisation fonctionnelle du parcours de traitement d' un patient en radiothérapie conciliant les objectifs de précision d'une modélisation détaillée aux impératifs de généricité garants d'une facilité de diffusion du modèle à différents services.

La méthodologie proposée s'appuie sur des hypothèses de structuration issues de l'ingénierie système et du génie cognitif et fait appel à l'utilisation systématique des « motifs de modélisation » et au principe «d'analyse des flux ».

L'application systématique de cette démarche de modélisation facilite la construction de modèles exhaustifs et structurés du parcours de traitement des patients dans un service de radiothérapie. Les écarts entre le modèle théorique et les activités réellement observées sont facilement identifiés, pouvant faire l'objet, le cas échéant, de mesures correctives. L'analyse de ces écarts devrait permettre à chaque service de cibler les voies d'amélioration propres à sa structure de soins et de prévenir l'occurrence d'évènements redoutés grâce à la diffusion d'une culture de sécurité.

Les hypothèses de modélisation issues de l'ingénierie sont applicables à la modélisation fonctionnelle du parcours de traitement des patients en radiothérapie. Les modèles produits sont suffisamment détaillés pour servir de base à une analyse dysfonctionnelle et, in fine, permettre la proposition et l'évaluation de barrières de prévention des risques.

Mots clés: Analyse de risques, Sécurité, modélisation fonctionnelle, motifs fonctionnels

\begin{abstract}
:
This document presents a systematic and structured approach for functional modeling for risk analysis in radiotherapy, aiming to reconcile the need on one hand for a method that can be applied generally and on the other hand for a method that provides a highly precise model.

The approach relies on new functional structuring patterns and flux analysis, derived from system engineering and knowledge engineering.

The method affords strong support for the development of detailed models of the patient's process through a department of radiotherapy. Lack of structure of the actual process in a particular department may be easily identified leading to the development of specific procedures for the improvement of security.

Modeling approach derived from engineering may be used for functional modeling for risk analysis in radiotherapy.
\end{abstract}

Keywords: Risk analysis, Safety, functional modeling, functional patterns.

\section{Introduction}

Afin de limiter les risques d'incidents et/ou accidents pouvant survenir au cours des traitements de radiothérapie, l'Organisation Mondiale de la Santé (OMS)[1]et l'Autorité de Sûreté Nucléaire (ASN)[2] ont édité des guides d'analyse de risques ayant pour objectif de sensibiliser chaque centre de soins aux démarches à mettre en œuvre pour aboutir à la définition de dispositifs de prévention ou de protection.

Ces démarches dédiées à la sûreté de fonctionnement reposent sur l'analyse précise du processus de mise en œuvre d'un traitement en radiothérapie[3-13] et s'apparentent aux pratiques industrielles concernant les systèmes techniques à risque[14-17]. Elles s'appuient généralement sur : 
- un modèle fonctionnel du système à risque qui décrit son fonctionnement, ses composants et les interactions entre ces composants,

une analyse dysfonctionnelle décrivant les différents modes de défaillance du système, leurs causes potentielles et leurs conséquences sur l'apparition d'événements indésirables,

des propositions de mesures ou dispositifs de prévention et de protection associés aux modes de défaillance.

L'application de ces démarches dans le domaine de la radiothérapie est confrontée, à l'instar des grands systèmes techniques complexes (nucléaire, transports terrestres ou aériens, ...) à une difficulté majeure relative au caractère sociotechnique de ces systèmes, un système sociotechnique étant défini comme «un ensemble composite de personnels, de matériels et de logiciels organisés pour que leur interfonctionnement permette, dans un environnement donné, de remplir les missions pour lesquels il a été conçu »[18, 19].

La préparation et la délivrance des traitements de radiothérapie externe relèvent :

d'une composante humaine et organisationnelle relative à la gestion d'un personnel médical multidisciplinaire comprenant les radiothérapeutes, les physiciens médicaux, les dosimétristes et les manipulateurs.

- d'une composante technique reposant sur des dispositifs médicaux de haute technologie pour l'acquisition de l'imagerie médicale (scanner), le calcul prévisionnel de la distribution de dose et son optimisation (consoles de dosimétrie) et la délivrance du traitement en conformité avec la prescription (accélérateurs linéaires).

L'analyse des incidents survenus à travers le monde sur les trente dernières années révèleque ce sont les facteurs humains et organisationnels qui sont à l'origine de la majorité d'entre eux[1, 19, $20]$.

L'obtention de modèles fonctionnels et dysfonctionnels dédiés à l'analyse des composantes humaine et organisationnelle de la radiothérapie est particulièrement difficile en raison du caractère multidisciplinaire de la spécialité qui fait appel à des niveaux de compétence professionnelle très variés[21]. De la qualité de ces modèles dépend pourtant toute démarche de prévention et de protection des risques. Face à cette difficulté, les modèles proposés en radiothérapie adoptent aujourd'hui deux stratégies complémentaires :

des études macroscopiques, comme les guides de l'ASN[2], de l'OMS[1] ou les travaux de Nascimento[13], présentent les grandes phases du traitement par radiothérapie, les principales catégories de problèmes associés à ces phases et des éléments généraux de prévention ; si elles constituent un cadre indiscutable à toute analyse de sécurité en radiothérapie, elles n'ont pas pour objet d'apporter des réponses pragmatiques à mettre en œuvre ;

des études détaillées[3, 4] développées pour un centre de soin donné et pour un dispositif de traitement particulier à l'intérieur de ce centre; ces études s'avèrent très couteuses en temps et peu diffusables d'un centre à l'autre, voire d'un dispositif à l'autre à l'intérieur du même centre.

Dans une démarche globale pour le développement d'un processus optimal d'amélioration de la sécurité en radiothérapie, nous proposons dans un premier temps les résultats de l'étape préliminaire relative à la modélisation fonctionnelle du parcours de traitement du patient. Ce travail a été mené avec le souci de concilier les objectifs de précision d'une modélisation détaillée avec des impératifs de généricité garants d'une facilité de diffusion du modèle à différents services. Nous proposons l'utilisation systématique des «motifs» de modélisation permettant d'une part, de structurer le modèle et d'autre part, de faciliter l'analyse des écarts entre le parcours de traitement réel et le modèle théorique.

Les modèles produits à l'aide de cette démarche doivent être suffisamment détaillés pour servir de base à une analyse dysfonctionnelle ultérieure et, in fine, permettre la proposition et l'évaluation de barrières de prévention et de protection.

\section{Matériel et Méthodes}

\subsection{Motif Bloc Système.}

L'une des clefs de l'ingénierie système est la représentation simplifiée du cycle d'ingénierie selon un modèle en forme de $\mathrm{V}$ constitué par une succession de blocs systèmes[22]. La figure 1 présente ce schéma de modélisation. La branche descendante du V (branche d'ingénierie) permet de passer du besoin à la réalisation alors que la branche ascendante (branche d'intégration) concerne les phases d'intégration et de validation.

Le lien entre les branches descendante et ascendante est assuré par la succession de blocs systèmes. Un bloc système se définit par son activité propre dans le processus global d'ingénierie. Chaque bloc système possède une activité qui dépend de celle des blocs précédents et impacte celle des blocs suivants. Deux types d'activités sont associés à chaque bloc :

une activité de spécification/conception qui s'intègre dans la branche descendante du modèle en V.

Le bloc système du niveau $\mathrm{N}$ transforme une exigence issue de l'activité du bloc système précédent $(\mathrm{N}+1)$ en une production qui définit une exigence pour le bloc système suivant $(\mathrm{N}-1)$.

une activité d'intégration/validation qui s'intègre dans la branche montante du modèle en V. Cette activité correspond à la validation de la production issue de l'activité de spécification/conception du bloc considéré.

Ce schéma de modélisation peut être appliqué au parcours de prise en charge des patients en radiothérapie (figure 1). Concernant la branche descendante du modèle en $\mathrm{V}$, elle correspond à la phase de planification du plan de traitement allant de la prescription (besoin) à la transcription des paramètres machines (réalisation) dans le système de vérification et d'enregistrement du traitement (Record and Verify software). La branche ascendante regroupe quant à elle l'ensemble des procédures de validation de cette phase de planification, procédures qui doivent être réalisées dans leur intégrité pour autoriser l'initiation du traitement.

Chaque bloc système peut être associé aux étapes successives de préparation du plan de traitement et fait appel aux compétences professionnelles propres à la spécialité de radiothérapie (radiothérapeutes, physiciens médicaux, dosimétristes et manipulateurs).

La figure 2a présente une vision schématique d'un bloc système et la figure $2 \mathrm{~b}$ propose une application du bloc système à l'étape «définition d'une balistique de traitement». L'activité de spécification/conception de ce bloc répond aux exigences définies par le bloc précédent « définition des volumes » et se définit par la mise en place adaptée d'un isocentre de traitement et le choix d'une balistique. produit:

L'activité de spécification/conception du bloc système considéré

- les exigences du bloc suivant intitulé «optimisation dosimétrique ».

- des paramètres utiles à la validation ultérieure de sa production. Ces paramètres définissent un «scénario de validation » qui sera utilisé lors de l'activité d'intégration/validation correspondante (branche montante du modèle en $\mathrm{V}$ ).

L'enchainement des activités des différents blocs système successifs le long de la branche descendante du modèle en $\mathrm{V}$ permet d'affiner le plan de traitement depuis la prescription médicale jusqu'à la transcription des paramètres de traitement. 
Cette première étape aboutit à une structuration macroscopique des différentes activités liées au parcours du patient. Il convient par la suite de détailler chacune de ces activités et de préciser les flux échangés entre celles-ci. Pour ces deux points, nous proposons d'utiliser respectivement :

- le motif actinomique «Préparation-Réalisation-Clôture » pour la description détaillée de chaque activité.

- $\quad$ et une taxinomie de typage des flux d'objets pour détailler les flux échangés entre les activités.

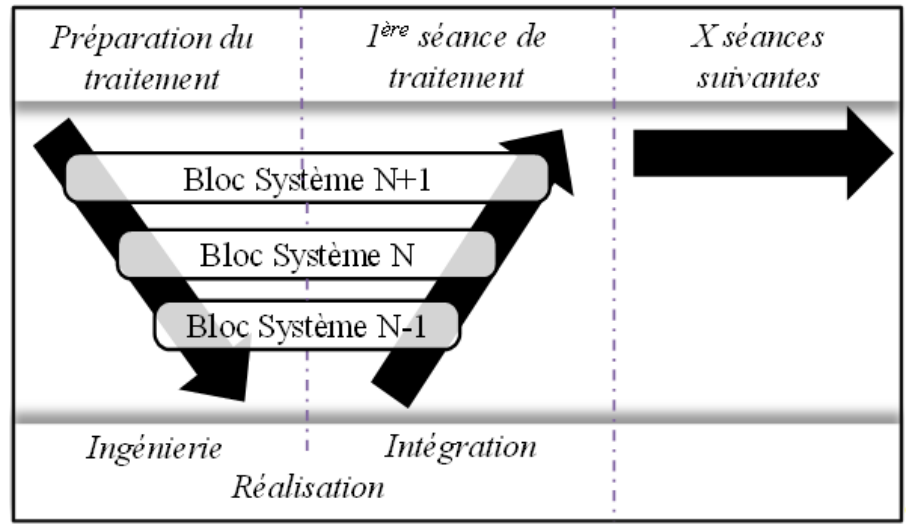

Figure 1 : Représentation schématique du cycle en Vd'ingénierie.

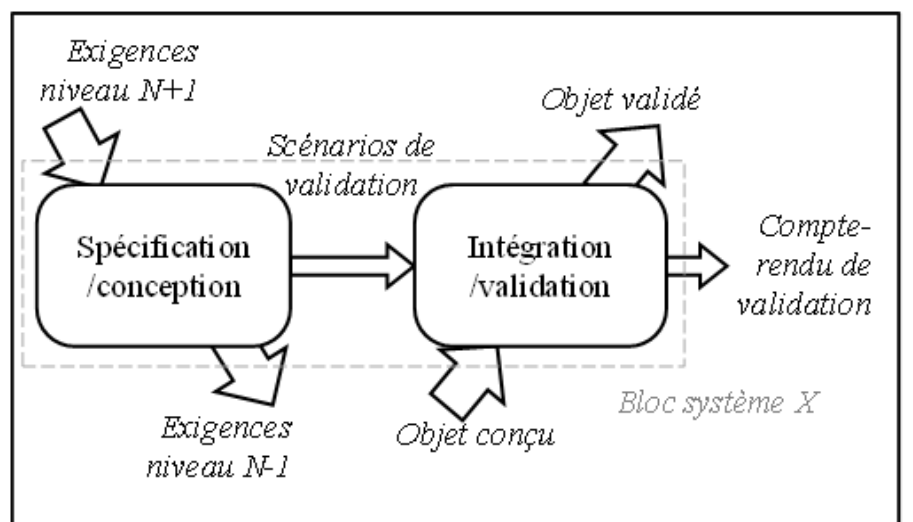

a)Vision schématique d'un bloc système.

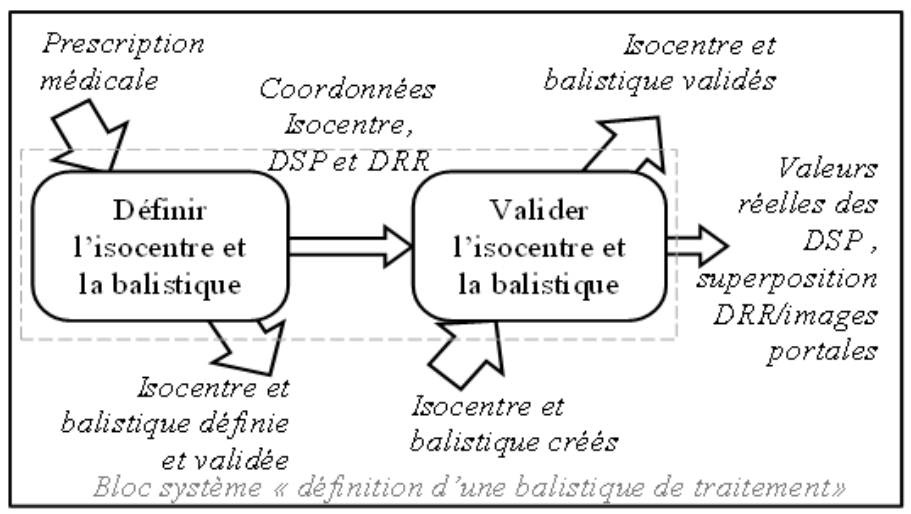

b) Application du bloc à l'étape « définition d'une balistique de traitement».

Figure 2 : Illustrations de l'utilisation du bloc système. DSP : Distance Source-Peau, DRR : Digital Reconstruction Radiograph.

\subsection{Motif actinomique « Préparation-Réalisation-Clôture ».}

Afin de structurer la connaissance des actions humaines impliquées dans la maintenance et la conduite des systèmes industriels, Léger [23]propose de classer en trois phases les actions humaines nécessaires à la réalisation d'une tâche. Cette structuration est issue de travaux en génie cognitif [24]sur le processus de changement organisationnel et se rapproche du concept de « quality gate » [25].

Les trois phases se décomposent ainsi :

- la phase de «Préparation» porte sur l'organisation, la spécification et la caractérisation de toutes les conditions nécessaires à l'exécution de l'activité ; elle vise à filtrer les flux d'entrées de l'activité au regard d'un référentiel d'exigences pour le métier considéré.

- la phase de «Réalisation » concerne la mise en œuvre et la coordination des moyens afin d'exécuter l'activité considérée;

- la phase de «Clôture » porte sur la vérification des travaux réalisés au cours de l'activité, l'analyse des informations collectées en cours et en fin de travaux, et les retours d'expériences; cette étape vise à s'assurer du bon déroulement de l'activité.

Cette structure «Préparation-Réalisation-Clôture » (P-R-C) est une séquence itérative, que l'on peut décliner depuis le niveau le plus macroscopique jusqu'aux niveaux les plus détaillés de la modélisation fonctionnelle. La figure 3 donne une vue schématique de ce motif structurant.

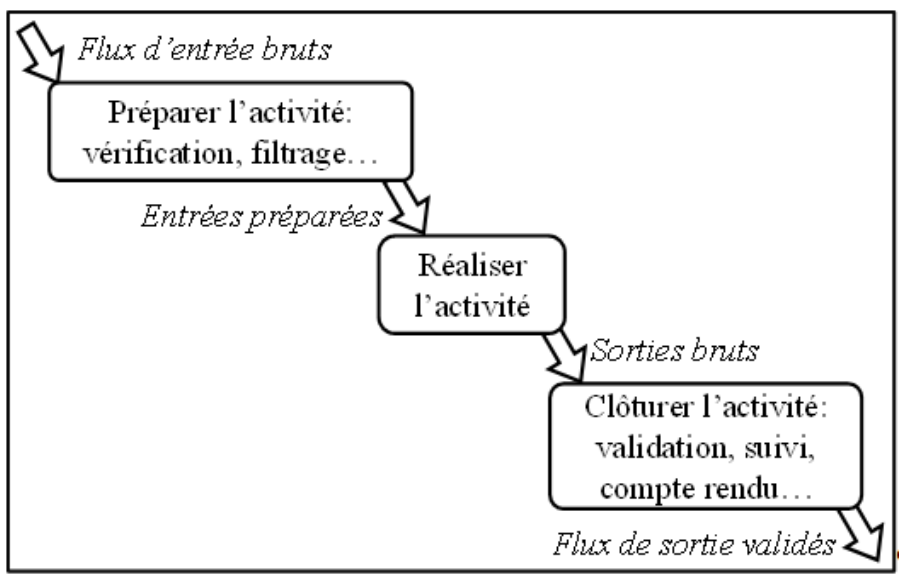

Figure 3: Schéma du motif actinomique $P-R-C$

En reprenant l'exemple du paragraphe précédent concernant le bloc système associé à la « définition d'une balistique de traitement », l'application du motif P-R-C conduit à décomposer :

- l'activité de spécification/conception (figure 4a) en une phase de «Préparation» pour valider la délinéation préalable des volumes par le médecin; une phase de «Réalisation » relative au choix d'un isocentre, de la balistique et de la conformation des champs; une phase de "Clôture » pour enregistrer la fin de l'activité et produire le scénario de validation.

- l'activité d'intégration/validation (figure 4b) par une phase de «Préparation» consistant à vérifier la disponibilité et la validité du «scénario de validation» qui comprend les informations DRR (Digital Reconstruction Radiograph) et DSP (Distance Source-Peau), une phase de «Réalisation » consistant à valider le positionnement de l'isocentre et la conformation des champs en utilisant les informations DSP et DRR vérifiées, et enfin une phase de «Clôture » consistant à enregistrer les décalages éventuels d'isocentre issus de la superposition des images portales $\left(0^{\circ}\right.$ et $\left.90^{\circ}\right)$ avec les DDR ainsi que les DSP réelles qui en découlent. 


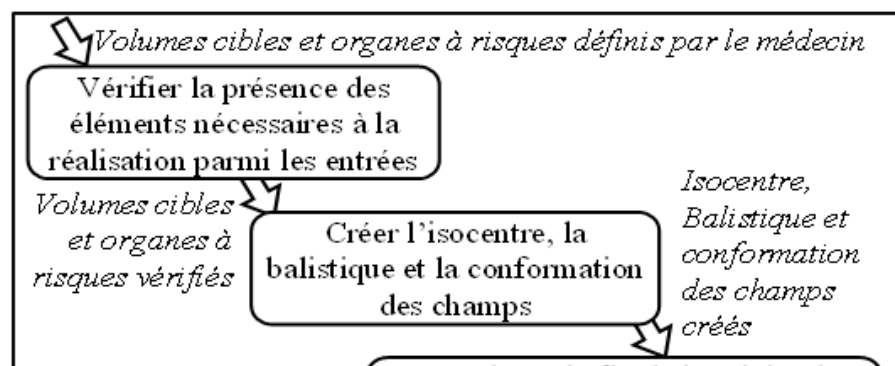

Enregistrer la fin de l'activité de réalisation, noter les coordonnées de l'isocentre, réaliser les DRR et DSP

Isocentre, balistique et conformation des champs validés, $Y$ coordonnées de l'isocentre, DRR et DSP créées

a)Décomposition en $P-R-C$ de l'activité de spécification/conception $d u$ bloc système " conception d'une balistique de traitement ».

Position de l'isocentre de traitement validé, Valeurs réelles des DSP et résultats de la superposition DRR/images portales $\Sigma$ Noter les DSP réelles, Imprimer les résultats de la superposition $\mathrm{DRR} /$ images portales

coordonnées de
l'isocentre,
DRR et DSP
vérifiées

Vérifier la présence et la cohérence des coordonnées de l'isocentre, des DRR et DSP

\section{$\$$ Coordonnées de l'isocentre, DRR et DSP}

b) Décomposition en $P-R-C$ de l'activité d'intégration/validation du bloc système " conception d'une balistique de traitement ».

Figure 4 : Schémas de la décomposition en P-R-C des activités du bloc système " conception d'une balistique de traitement».

\subsection{Typage des flux d'objets}

Pour structurer les flux échangés entre les activités identifiées précédemment, nous proposons la typologie suivante [26, 27] :

- «Devoir-Faire»(DF) : il définit la finalité de l'activité (exemple: définition d'une balistique) en passant d'un objectif (entrée) à une réalisation (sortie).

- «Vouloir-Faire »(VF) : c'est le flux déclenchant la réalisation de l'activité. Il correspond principalement à un signal d'initiation de l'activité (exemple : la production de volumes validés par le médecin autorise le déclenchement de l'étape suivante de définition de la balistique). Il peut s'agir également d'un flux de cadencement imposé (exemple : les volumes doivent être définis 5 jours avant la première séance).

- «Savoir-Faire»(SF) : ce flux regroupe l'ensemble des connaissances/informations requises à la réalisation de 1'activité (exemple: protocoles, procédures et guides de réalisation).

- «Pouvoir-Faire » (PF) : ce flux correspond aux ressources humaines ou matérielles disponibles pour la réalisation de l'activité. Au cours de l'activité, ces ressources sont éventuellement transformées (exemple: des consommables comme certains matériels de contention sont dégradés en matériel usagé) ou produites (exemple: les scénarios de validation). Pour cette raison, le flux PF est observé en entrée et en sortie de l'activité.

La figure 5 illustre graphiquement cette typologie de flux. Les flux de «Savoir-Faire » et de «Pouvoir-Faire » sont, par définition, les points d'entrée des facteurs organisationnels d'un service qui influent sur le parcours de prise en charge des patients.

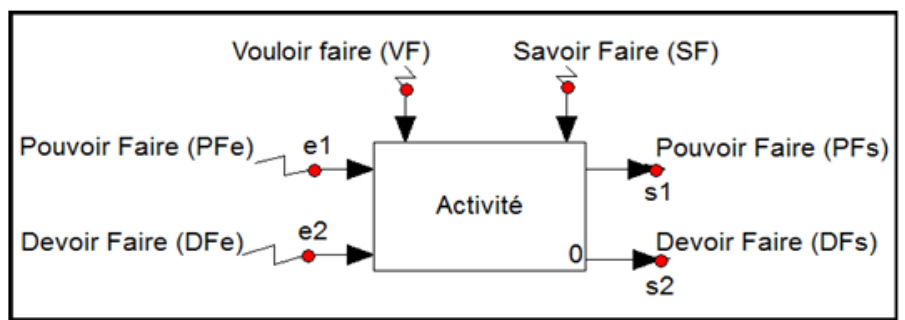

Figure 5: Typage des flux d'objets. e : entrée, $s$ : sortie.

\subsection{Démarche de modélisation fonctionnelle structurée.}

Les différents «motifs» de modélisation définis dans les paragraphes précédents (blocs systèmes; P-R-C; typage des flux) servent de base à un questionnement systématique, permettant de construire, de manière rationnelle et itérative, l'ensemble des modèles fonctionnels du parcours suivi par le patient.

La démarche de modélisation proposée repose sur 3 phases principales : l'identification des activités de spécification/conception, l'identification des activités d'intégration/validation correspondantes et enfin la décomposition de l'ensemble des activités identifiées selon le motif P-R-C. Cette dernière phase est reproduite pour chaque niveau de décomposition selon la granularité et le niveau de détail souhaités.

La description la plus exhaustive possible des activités de spécification/conception identifiées passe par la réponse systématique aux questions suivantes:

- à quelle exigence l'activité répond-t-elle: quel est le «Devoir-Faire» en entrée issu des exigences de sortie générées par l'activité précédente (niveau $\mathrm{N}+1$ ) ?

- quel objet l'activité doit-elle créer ou modifier : quel est le «Devoir-Faire» en sortie produit par cette activité et qui définira les exigences pour l'activité suivante (niveau N-1)?

- quel flux spécifique (autorisation d'exécution, consignes, ...) déclenche l'activité : quel est le « Vouloir-Faire»?

- de quel objet l'activité a-t-elle besoin pour se réaliser et cet objet est-il modifié au cours de l'activité: quel est le «Pouvoir-Faire » en entrée et son éventuelle modification en sortie?

- quels sont les moyens de contrôles, ou objets spécifiquement produits pour s'assurer que l'objet conçu est conforme à l'exigence auquel répond l'activité étudiée : quel scénario de validation l'activité va-t-elle produire («Pouvoir-Faire» en sortie) ?

- quel guide, protocole ou procédure est à disposition pour contribuer à la bonne réalisation de l'activité : quel est le «Savoir-Faire » disponible?

Cette démarche systématique permet de faciliter la construction des modèles en levant les ambiguïtés et en identifiant les éventuels manques de structuration fonctionnelle du parcours patient.

Selon les préceptes du bloc système précédemment présentés, il convient d'associer à chaque activité de spécification/conception identifiée, un ensemble d'activité d'intégration/validation. Ces activités 
réalisées avant ou lors des premières séances de traitement peuvent être identifiées sur la base du questionnement systématique suivant :

- quelles sont les modalités de validation de l'activité de spécification/conception considérée : description du « scénario de validation »?

- comment cette activité de validation est-elle consignée: description du « compte-rendu de validation »?

A nouveau, cette démarche systématique permet de faciliter la construction des modèles en levant les ambiguïtés et en identifiant les éventuels manques de structuration des procédures de contrôle et de validation.

Ces deux étapes aboutissent à un modèle fonctionnel contenant les activités de spécification/conception liées à la planification du traitement (activités à gauche de chaque bloc système, le long de la branche descendante du modèle en $\mathrm{V}$ ), et les activités d'intégration/validation de cette planification (activités à droite de chaque bloc système, le long de la branche ascendante du modèle en V).

La modélisation se poursuit en appliquant les principes du motif $\mathrm{P}$ $\mathrm{R}-\mathrm{C}$ à chacune des activités identifiées. Cette démarche s'appuie sur les deux questions suivantes :

- quelles sont les étapes de préparation nécessaires avant la réalisation de l'activité étudiée ? Il peut s'agir d'opérer des transformations préalables de certains flux d'entrée pour les mettre en conformité avec les besoins de l'activité (exemple : changement de référentiels, d'unités de traitements, ...) ou des opérations de vérification des flux d'entrée (exemple : vérification de l'identité du patient, ...).

- quelles sont les étapes de clôture imposées à l'issue de la réalisation de l'activité ? Il peut s'agir d'opérations de rangement, de remise en état des matériels utilisés, de consignation de compte-rendu d'exécution, ou de vérification du bon déroulement de l'activité réalisée.

La démarche de structuration selon le motif P-R-C peut être appliquée de manière itérative à chaque niveau hiérarchique de la modélisation. Le point d'arrêt de l'étude de ces fonctions apparaît lorsque le modélisateur atteint un niveau de détail suffisant à l'étude des dysfonctionnements qu'il souhaite mener. La modélisation ne pourra pas aller au-delà de la description des fonctions élémentaires du système, qui peuvent correspondre à :

- une fonction supportée par un système technique « insécable » (le support patient, un laser...),

- une action simple (noter le paramètre A, marquer la projection du laser $\mathrm{Z}$ sur le patient...),

une action supportée par un humain précis (Manipulateur A ou B, Physicien A ou B...),

- lorsque plus aucune des sous activités étudiées et modélisées ne nécessite de préparation ou de clôture.
Lorsque l'ensemble des activités de spécification/conception et d'intégration/validation liées à l'élaboration du plan de traitement seront modélisées avec le niveau de précision requis, la même démarche de modélisation systématique et itérative (identification des réalisations principales, identification des flux, application du motif $\mathrm{P}$ $\mathrm{R}-\mathrm{C}$ ) pourra être appliquée au processus de réalisation des séances de traitement.

\section{Résultats}

La méthodologie présentée dans ce document a été appliquée à la modélisation du parcours patient dans le service de radiothérapie du Centre Alexis Vautrin.

Les figures sont réalisées en langage Structured Analysis and Design Technique (SADT) à l'aide du logiciel Orchis (GeenSoft). En comparaison des autres méthodes disponibles, SADT est remarquable par la simplicité et la concision de son formalisme graphique (boites et flèches) facilitant la lecture des modèles complexes[28]. En revanche, le point faible de SADT concerne le manque d'identification des flux entrant/sortant pour chaque activité. Cette lacune est corrigée par la méthodologie proposée qui prévoit une recherche systématisée des flux de chaque activité.

La démarche de modélisation a été appliquée dans un premier temps à la phase d'élaboration du plan traitement. Différents blocs systèmes ont été identifiés, tous supportés par un type de profession différent. Le premier bloc est supporté par le radiothérapeute qui a en charge la définition des cibles et la prescription des contraintes dosimétriques. Les deux blocs suivants sont supportés par un physicien médical et/ou un dosimétriste qui définit la balistique et optimise la distribution de la dose. Le dernier bloc relève de la responsabilité du physicien médical et correspond à la transcription des paramètres issus des activités précédentes en des paramètres techniques directement interprétables par l'appareil de traitement. La figure 6 présente le modèle fonctionnel SADT de ces différents blocs.

La figure 7 présente les modèles SADT appliqués à l'étape de réalisation des séances de traitement. Dans le cadre supérieur de la figure, l'activité étudiée est la fonction « réaliser l'irradiation» $\left(\mathrm{n}^{\circ} 2\right)$. Les fonctions correspondantes de préparation $\left(\mathrm{n}^{\circ} 1\right)$ et de clôture $\left(\mathrm{n}^{\circ} 3\right)$ ainsi que tous les flux présentés sont issus de l'application de la démarche systématique de modélisation. Le cadre inférieur de la figure présente la décomposition de l'activité «préparer l'irradiation » $\left(\mathrm{n}^{\circ} 1\right)$ qui nécessite elle-même des fonctions de préparation (vérification de la présence des dossiers et du matériel appartenant au patient, ainsi que de la présence et de l'identité du patient), et des fonctions de clôture (contrôle de la position du patient installé avec son matériel de contention dans la salle de traitement, évacuation et verrouillage de la salle de traitement). 


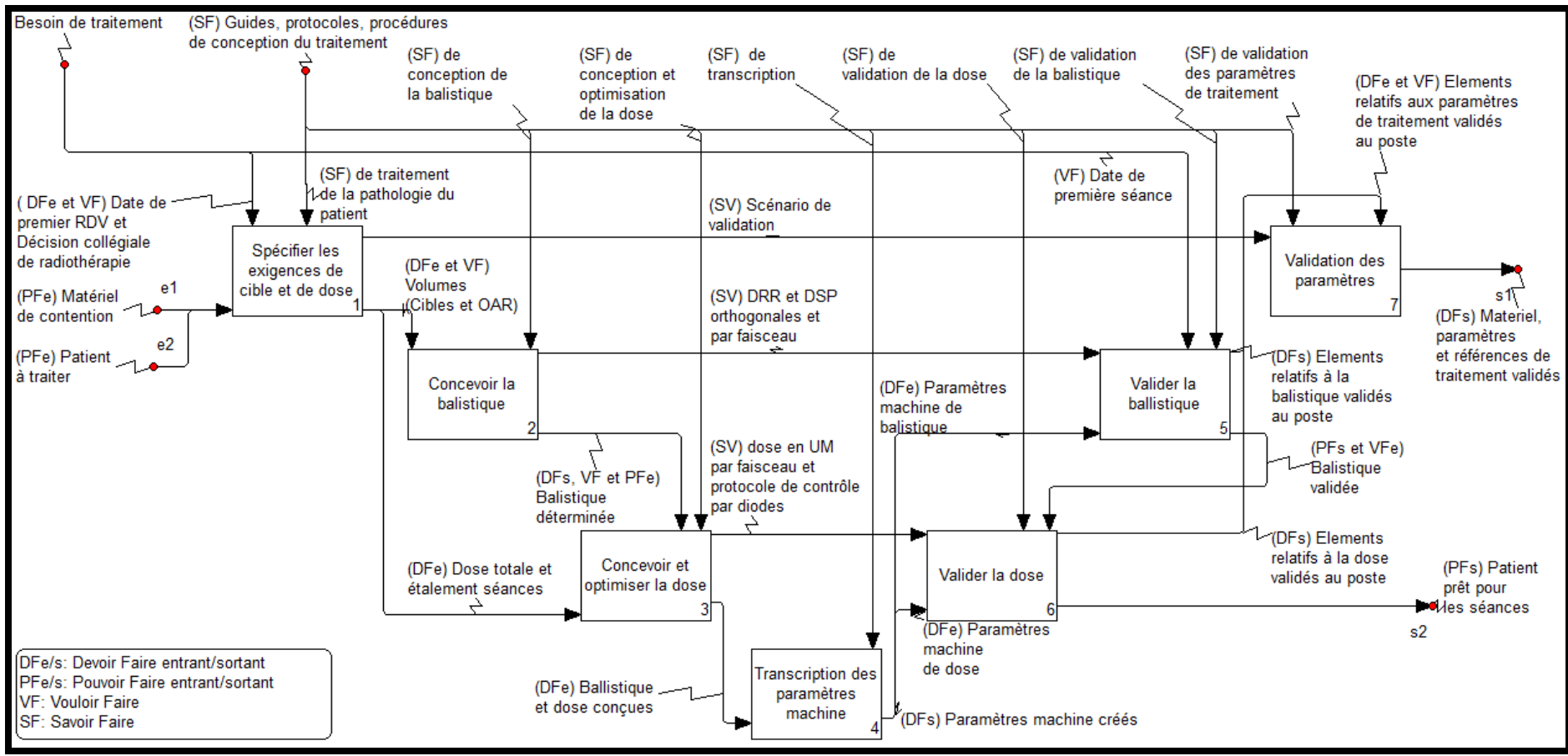

Figure 6: Modèle SADT de la phase de conception/validation des paramètres de traitement.
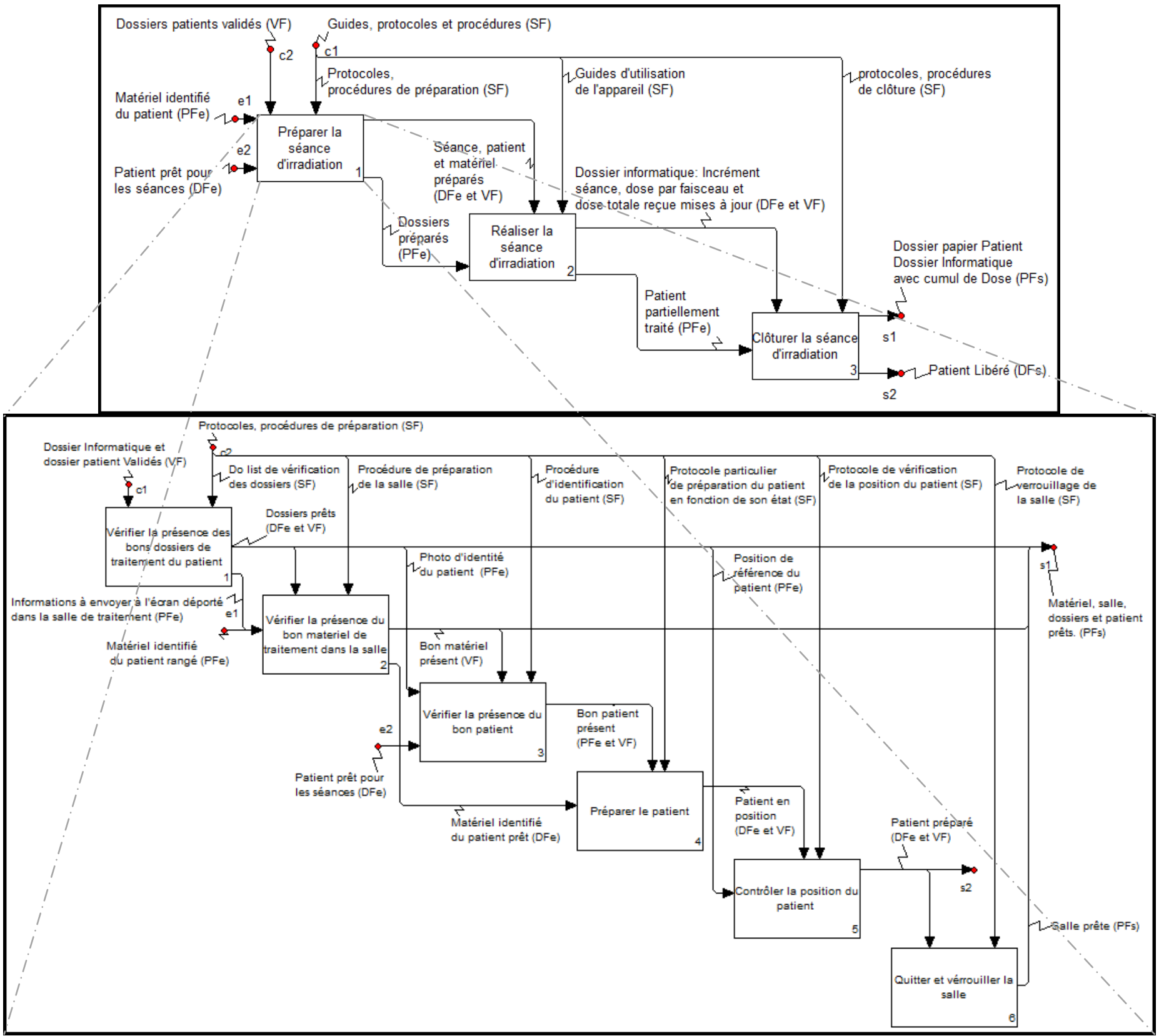


\section{Discussion}

Des institutions telles que l'ASN[2] ou l'OMS[1] ou encore les travaux de Nascimento[13] sur la culture de sécurité en radiothérapie proposent une description fonctionnelle macroscopique du parcours suivi par le patient en radiothérapie. Les modèles présentés sont limités à un nombre restreint d'activités dont les productions et les ressources sont décrites. L'objectif de ces guides est l'évaluation de risques " génériques 》, basée sur une analyse dysfonctionnelle (ASN) ou sur une revue de littérature traitant des incidents de radiothérapie (OMS). Cette démarche à l'intérêt de ne pas dépendre d'un centre de soin particulier ou d'une technique de traitement spécifique. En revanche, ces études sont limitées à l'identification de risques ou de dysfonctionnements génériques qu'il est peu aisé de combattre sans informations complémentaires.

La construction d'un modèle fonctionnel détaillé apparait comme un préalable indispensable à une analyse dysfonctionnelle précise dont l'objectif est d'identifier les modes de défaillance, leurs causes et leurs conséquences pour, au final, définir et valider des moyens de prévention et de protection des risques.

Cependant, ces modèles détaillés sont d'élaboration difficile et fastidieuse. Ces études sont couteuses en temps et requièrent l'expertise des professionnels impliqués. Par ailleurs, les modèles sont développés par un centre de soin donné, selon une méthodologie qui lui est propre et parfois pour un dispositif de traitement particulier, ce qui limite les possibilités de diffusion d'un service à l'autre.

$\mathrm{Au}$ regard de ces difficultés, nous proposons l'élaboration d'un modèle fonctionnel basé sur l'utilisation systématique des «motifs » de modélisation. Cette approche nous semble présenter un double intérêt:

les motifs proposés structurent et systématisent la démarche de modélisation et concilient des objectifs de généricité et de simplicité de mise en œuvre avec des objectifs de précision.

- l'application systématique et itérative des motifs attire l'attention du modélisateur sur les écarts existants entre un modèle théorique exhaustif et la réalité des activités observées lors du parcours d'un patient. Ces écarts ne constituent pas nécessairement un facteur de risque lorsqu'ils sont justifiés et documentés.

Les motifs de structuration utilisés sont issus de l'Ingénierie Système (Bloc système), du génie cognitif (Structuration P-R-C) et de la sémiotique (typage des flux). Ces disciplines, les concepts utilisés et les motifs proposés ont démontrés leur utilité dans le domaine de la sûreté industrielle à différents niveaux :

- Le cycle en $\mathrm{V}$ et la vision en blocs systèmes sont employés dans le domaine industriel pour la conception de systèmes complexes [22];

L'actinomie P-R-C développé par Vogel [24]a déjà été utilisée pour modéliser, analyser et quantifier la sûreté des taches de conduite ou de maintenance de grands systèmes industriels [23]

- La taxinomie des flux est issue de la sémiotique et du génie systémique [27], et est utilisée régulièrement pour l'étude des systèmes complexes [26] ;

Notre démarche a montré que ces différents motifs, issus du monde de l'ingénierie, sont applicables à la modélisation fonctionnelle $\mathrm{du}$ parcours de traitement des patients en radiothérapie. Leur application itérative et systématique facilite l'obtention de modèles détaillés. Cette démarche ne fait pas obstacle à la prise en compte des spécificités fonctionnelles liées à chaque centre puisqu'elle repose sur l'expertise des professionnels impliqués. Néanmoins, sa systématisation facilite et structure le recueil de cette expertise.

La génération d'un modèle détaillé basé sur l'utilisation récurrente des «motifs» de modélisation permet d'identifier les écarts entre les préconisations de structuration contenues dans ces «motifs » et la réalité du processus de prise en charge du patient. Par exemple, l'utilisation des blocs systèmes souligne le couplage fort qui devrait exister entre chaque activité de conception avec des activités de validation dédiées. L'analyse d'une activité spécifique peut mettre en évidence, sur le système réel, le défaut d'identification d'un type de flux lié à cette activité. Cette absence peut être justifiée mais devra, dans ce cas, être documentée. A l'inverse, cette absence peut révéler une fragilité devant faire l'objet d'analyses approfondies voire de corrections. De la même manière, si la symétrie théorique du motif P-R-C n'est pas observée pour une activité, l'absence de préparation et/ou de clôture pourra être justifiée et documentée, ou au contraire faire l'objet d'une procédure corrective.

Ainsi, chaque établissement peut mesurer l'écart entre ses pratiques réelles et le modèle théorique contenu dans les «motifs » de structuration, et justifier l'écart par des solutions alternatives. A titre d'exemple, pour l'activité « réaliser la séance d'irradiation ", la phase de préparation intègre une activité de vérification de la forme du faisceau qui doit être assurée, selon le «motif» théorique, avant chaque séance d'irradiation et qui, dans la pratique, est remplacée par une vérification de la forme du faisceau lors de la première séance de traitement et un contrôle périodique du collimateur.

Ces différents exemples illustrent comment l'utilisation systématique des «motifs » de modélisation génère une première identification de certains modes de défaillance et des procédures à mettre en œuvre pour les prévenir. Néanmoins, seule une analyse dysfonctionnelle complète assurera l'identification exhaustive de ces modes de défaillance pour permettre, au final, l'élaboration d'un plan efficient de prévention et de protection des risques.

\section{Conclusion}

L'application des «motifs» de modélisation issus de l'ingénierie système à l'analyse du parcours des patients en radiothérapie facilite la construction d'un modèle détaillé grâce à une démarche systématique et structurée. Cette démarche offre également la possibilité de mesurer et documenter les écarts entre les constructions théoriques proposées et les activités réellement observées. L'analyse de ces écarts devrait permettre à chaque centre de cibler les voies d'amélioration propres à sa structure de soins et de prévenir l'occurrence d'évènements redoutés grâce à la diffusion d'une meilleure culture de sûreté.

La modélisation fonctionnelle présentée dans ce document est un préalable à une démarche d'analyse dysfonctionnelle qualitative, de type AMDE (Analyse des Modes de Défaillances et de leurs Effets) ou HazOp (Hazard and Operability). Comme la modélisation fonctionnelle, l'analyse dysfonctionnelle fait face à 2 écueils: une analyse trop macroscopique ne permet pas l'identification précise des moyens à mettre en œuvre pour prévenir les causes de défaillance, alors qu'une analyse détaillée assure l'élaboration de barrières efficientes mais reste de réalisation difficile. La définition d'une démarche d'analyse dysfonctionnelle systématique basée sur la modélisation 
fonctionnelle présentée dans ce travail et visant à s'affranchir de ces 2 écueils, fait l'objet de la publication suivante.

Références :

1. World Health Organization (WHO). Radiotherapy Risk Profile. Switzerland: WHO Press; 2008.

2. Autorité de Sureté Nucléaire (ASN). Guide de l'ASN numéro 4: Guide d'auto-évaluation des risques encourus par les patients en radiothérapie externe; 2009. Consultable sur: http://www.asn.fr/.

3. Scorsetti M, Signori C, Lattuada P, Urso G, Bignardi M, Navarria $P$, et al. Applying failure mode effects and criticality analysis in radiotherapy: lessons learned and perspectives of enhancement. Radiother Oncol. 2010;94:367-74.

4. Nguyen TD, Devie I, Heusghem M, Gaillot-Petit N, Loiseau M. [Cartography and risk management in radiotherapy: A collaborative work of the department of radiotherapy and the department of quality and risk management at the Jean-Godinot Institute]. Cancer Radiother. 2010;14:24-8.

5. Aigle D, Sobczyk C, Androni ML, Peiffert D, Beckendorf V, Marchesi V, et al. [Safety and quality assurance management in radiation oncology "what is the role for qualiticians?": example of the Centre Alexis Vautrin]. Cancer Radiother. 2009;13:455-7.

6. Arnold A, Delaney GP, Cassapi L, Barton M. The use of categorized time-trend reporting of radiation oncology incidents: a proactive analytical approach to improving quality and safety over time. Int J Radiat Oncol Biol Phys. 2010;78:1548-54.

7. Bissonnette JP, Medlam G. Trend analysis of radiation therapy incidents over seven years. Radiother Oncol. 2010;96:139-44.

8. Debouck F, Rieger E, Petit H, Noel G, Ravinet L. [ORION®: A simple and effective method for systemic analysis of clinical events and precursors occurring in hospital practice.]. Cancer Radiother 2011; doi:101016/jcanrad201112002

9. Lartigau E, Vitoux A, Debouck F. [Crex and Orion analysis in radiation oncology: towards a mutualisation of corrective actions]. Cancer Radiother. 2009;13:458-60.

10. Mazeron JJ, Mornex F, Eschwege F, Lartigau E, Bolla M. [Increasing quality and safety of external radiation treatments: actions of the French Society of Radiation Oncology (SFRO)]. Cancer Radiother. 2008;12:601-5.

11. Talandier D, Tajahmady AT, Woynar S. [Radiotherapy safety: MEAH evaluation at three years]. Cancer Radiother. 2009;13:461-5.

12. Aloui S. Contribution à la modélisation et l'analyse du risque dans une organisation de santé au moyen d'une approche système. Docotrat de l'Ecole des Mines de Paris; 2007. Consultable sur: http://hal.archives-ouvertes.fr/tel-00204007/.

13. Nascimento A. Produire la santé, produire la sécurité : développer une culture collective de sécurité en radiothérapie. Docotorat d'Ergonomie du Conservatoire National des Arts et
Métiers; 2010. Consultable sur: http://tel.archives-ouvertes.fr/tel$00453447 / \mathrm{fr} /$.

14. Institut de Radioprotection et de Sureté Nucléaire (IRSN). Améliorer la sécurité des traitements en radiothérapie en développant une culture de sûreté; 2008. Consultable sur: http://www.irsn.fr/.

15. Institut National de l'Environnement Industriel et des Risques (INERIS). Rapport d'étude $\mathrm{N}^{\circ}$ DRA-08-95403-01561B. Evaluation des performances des Barrières Techniques de Sécurité (DCE DRA-73). Evaluation des Barrières Techniques de Sécurité - Oméga 10; 2008. Consultable sur: http://www.ineris.fr/.

16. Institut National de l'Environnement Industriel et des Risques (INERIS). Rapport d'étude N DRA-09-103041-06026B. Démarche d'évaluation des Barrières Humaines de Sécurité Oméga 20. Programme 181-DRA-77 : Maitrise des risques accidentels par les dispositions technologiques et organisationnelles; 2009. Consultable sur: http://www.ineris.fr/.

17. Francois $\mathrm{P}$, Lartigau E. [Risk analysis in radiotherapy]. Cancer Radiother. 2009;13:574-80.

18. Meinadier JP. Ingénierie et intégration des systèmes. Londres: Hermes Science Publishing Ltd; 1998.

19. Parmentier G. [Quality \& Safety in radiotherapy: advocacy for a professional strategy]. Cancer Radiother. 2008;12:584-600.

20. Peiffert D, Simon JM, Eschwege F. [Epinal radiotherapy accident: passed, present, future]. Cancer Radiother. 2007;11:30912 .

21. Sheard S. Definition of the Sciences of Complex Systems. In: INSIGHT vol. 9 (1). Seattle, WA: INCOSE.

; 2006. Consultable sur: http://www.incose.org/

22. afis.fr [site internet]. Association Française d'Ingéniérie Système. Consultable sur: http://www.afis.fr/.

23. Léger A, Weber P, Levrat E, Duval C, Farret R, Lung B. Methodological developments for probabilistic risk analyses of socio-technical systems. Journal of Risk and Reliability. 2009;223:333-45. Consultable sur: http://pio.sagepub.com/.

24. Vogel C. Génie cognitif. Paris: Masson; 1988.

25. Giebel M, Essmann H, Preez ND, Jochem R. Improved innovation through the integration of Quality Gates into the Enterprise and Product Lifecycle Roadmaps. CIRP Journal of Manufacturing Science and Technology. 2009;1:199-205.

26. Mayer F. Contribution to manufacturing engineering: Application to pedagogical engineering within a CIME centre. Doctorat de l'université Henri Poincaré de Nancy; 1995.

27. Coquet JC. Le discours et son sujet: Essai de grammaire modale. Paris: Méridiens Klincksieck; 1989.

28. Trilling L, Besombes B, Chaabane S, Guinet A. Rapport de recherche sur le projet hrp2. Investigation et comparaison des méthodes et outils d'analyse pour l'étude des systèmes hospitaliers; 2004. Consultable sur: http://agbo.insalyon.fr/ressources/fichiers/Modélisation_des_pratiques.pdf. 